OPEN ACCESS

Citation: V. Guerrini (2021) Donne immigrate, estremismi e radicalizzazione. Tra rischio di vulnerabilità e opportunità di divenire costruttrici di comunità. Rief 18 , 2: pp. 131-145. doi: https://doi. org/10.36253/rief-10670.

Copyright: (c) 2021 V. Guerrini. This is an open access, peer-reviewed article published by Firenze University Press (https:// oaj.fupress.net/index.php/rief) and distributed under the terms of the Creative Commons Attribution License, which permits unrestricted use, distribution, and reproduction in any medium, provided the original author and source are credited.

Data Availability Statement: All relevant data are within the paper and its Supporting Information files.

Competing Interests: The Author(s) declare(s) no conflict of interest.

\section{Donne immigrate, estremismi e radicalizzazione. Tra rischio di vulnerabilità e opportunità di divenire costruttrici di comunità}

\author{
Valentina Guerrini ${ }^{1}$
}

\section{Abstract}

Il contributo affronta la complessa questione dell'estremismo e della radicalizzazione dal punto di vista della differenza di genere e, in particolare, del genere femminile. Infatti, tali problematiche sono per lo più associate al genere maschile e vi sono ancora pochi studi, soprattutto nel contesto nazionale, sulle forme di radicalizzazione al femminile. I primi studi e report a livello europeo evidenziano una partecipazione crescente delle donne nelle organizzazioni terroristiche negli ultimi anni e la forte presenza di forme di discriminazione e stereotipi sessisti all'interno delle organizzazioni stesse. Tuttavia, talvolta l'adesione a un'organizzazione terroristica può rappresentare per le donne, soprattutto inizialmente, un modo per sentirsi autonome e potersi riscattare da una situazione di pressione, discriminazione e violenza subita. Un'attenzione particolare viene dedicata alle giovani e alle donne musulmane perché maggiormente a rischio di discriminazioni ed esclusione sociale, e al ruolo delle madri immigrate come mediatrici di due culture e facilitatrici nel processo di inclusione sociale delle giovani generazioni, soprattutto se supportate da politiche e modelli educativi basati sull'inclusione, sul riconoscimento della differenza come valore, sull'accettazione dell'altro, sul dialogo e sul rispetto. A questo proposito, il contributo presenta i risultati di una ricerca condotta tra studenti e studentesse della scuola secondaria, per indagare sulle dinamiche di inclusione tra pari e sulle criticità connesse al periodo adolescenziale.

Parole chiave: radicalizzazione, giovani, donne, famiglia, discriminazione.

\section{Abstract}

This paper addresses the complex issue of extremism and radicalization from the perspective of gender difference and, in particular, the female gender. In fact, these issues are mostly associated with the male gender and there are still few studies, especially in the national context, on female forms of radicali-

${ }^{1}$ Assegnista di ricerca in Pedagogia generale e sociale presso il Dipartimento di Formazione, Lingue, Intercultura, Letterature e Psicologia (FORLILPSI) dell'Università degli Studi di Firenze. 
zation. The first studies and reports at European level highlight an increasing participation of women in terrorist organizations in recent years and the strong presence of forms of discrimination and sexist stereotypes within the organizations themselves. However, sometimes joining a terrorist organization can represent for women, especially initially, a way to feel autonomous and be able to redeem themselves from a situation of pressure, discrimination and violence suffered. Particular attention is devoted to young people and Muslim women because they are more at risk of discrimination and social exclusion and to the role of immigrant mothers as mediators of two cultures and facilitators in the process of social inclusion of younger generations, especially if supported by policies and educational models based on inclusion, recognition of difference as a value, acceptance of the other, dialogue, and respect. In this regard, the contribution presents the results of a research conducted among secondary school students to investigate the dynamics of inclusion among peers and critical issues related to the adolescent period.

Keywords: radicalisation, female youth, women, family, discrimination.

\section{Il problema dei comportamenti estremisti e della radicalizzazione nell'età giovanile, oggi}

Ladolescenza e, in parte oggi anche la giovinezza, rappresentano fasi estremamente complesse, difficili e molto significative nel processo di costruzione dell'identità di ogni individuo. Oggi più che mai, «nella società della Complessità, del disincanto della Flessibilità e di profonde Derive di Senso, vivere l'adolescenza si è fatto un iter ancora più inquieto e complicato" (Cambi, 2018, p. 9)2. Conseguentemente, anche i disagi adolescenziali e giovanili, visto che l'adolescenza si è socialmente allungata anche a causa della protratta dipendenza economica dalla famiglia, negli ultimi decenni sono incrementati e hanno assunto connotazioni diverse.

Come scrive Barone, l'adolescenza è per antonomasia il tempo della crisi e il luogo dell'ambiguità (2009). Anche il linguaggio definitorio delle scienze bio-mediche e psicologiche risulta troppo parziale per restituire la complessità del «sistema adolescenza» (ivi, p. 30). Un fenomeno spesso atteso da genitori e insegnanti come un «cataclisma» (ivi, p. 86) difficilmente gestibile; Mancaniello parla di «adolescenza come catastrofe» (2002, passim; 2018, passim) considerando tale fase come una condizione di crisi-trasformazione e di costruzione di un nuovo assetto.

Secondo gli studi di Erikson, sugli stadi di sviluppo (1980), all'adolescenza viene fatto corrispondere il complesso compito di sviluppo dell'identità con il possibile doppio esito di affermazione o confusione. Inoltre, negli ultimi anni è accresciuta, tra i giovani in particolare, ma non solo, la necessità di forme di partecipazione civica che trovano nei social media nuove possibilità di espressione: iniziative nate sulla Rete o trasferite e potenziate in Rete. Queste tendenze denotano l'esigenza dei soggetti, nella modernità avanzata, di conciliare individualismo e collettivismo (Baumann, 2005, trad. it. 2008), insieme al desiderio di affermare la propria identità soggettiva, coltivando interessi personali, e al contempo di condividere sentimenti, senso di solidarietà, di sentirsi parte di un "noi", entrando in una dimensione comunitaria.

Nella società odierna, caratterizzata dalla mobilità, dalla fluidità e dalla coesistenza di molteplici differenze (etniche, culturali, religiose, di genere, di orientamento sessuale) diventa estremamente importante conoscere e capire le dinamiche associative e partecipative dei giovani, in particolare degli adolescenti, in quanto rappresentano un canale privilegiato per l'acquisizione di una identità più stabile. Allo stesso modo, è necessario saper intercettare i primi segnali d'allarme di forme di esclusione sociale e devianza, affinché non degenerino in comportamenti

${ }^{2}$ Maiuscole e corsivi nella citazione in oggetto sono dell'Autore, N.d.R. 
estremisti e affinché questi giovani possano essere aiutati a inserirsi più armonicamente nella propria comunità.

Se oggi, vi è una maggiore possibilità di sviluppo del sé, libertà di esplorazione, scelta di valori e di legami, l'altra faccia della medaglia è che questo può portare a un senso di disorientamento, individualismo, relativismo e dissoluzione di legami (Besozzi, 2009). In particolare, per quanto riguarda i/le giovani non italiani/e possono emergere problematiche, conflitti e difficoltà di integrazione tra la cultura di origine e quella di arrivo, con la necessità urgente di ricomposizione di una varietà e pluralità di esperienze.

Molte ricerche sulle cosiddette "seconde generazioni" rilevano maggiori forme di disagio tra questi ragazzi e ragazze dovuti proprio alla mancanza del senso di appartenenza, alle difficoltà nella costruzione della propria identità, al fatto di essere talvolta stigmatizzati o discriminati per vari motivi che a seconda della situazione può essere il colore della pelle, la religione, la difficoltà a parlare Italiano, non avere le risorse economiche per poter condurre uno stile di vita come i compagni italiani (Bolognesi 2018; Dusi, 2017; D'Ignazi 2008, Fiorucci, Catarci, 2015; Guerrini, 2018; Silva, 2015, 2017).

L'aumentare delle disuguaglianze e dei conflitti sociali costituisce un terreno fertile per la radicalizzazione delle posizioni, che in alcune circostanze può esprimersi in forma violenta con modalità e intensità diverse: in molti casi si tratta di violenza verbale o discorsi d'odio, in altri casi si traduce in azioni discriminatorie e, nei casi più gravi, assume forme estreme che attentano alla vita stessa delle persone. L'estremismo violento, che non ha confini e colpisce tutte le società, coinvolge in particolare i giovani, che rappresentano il principale bersaglio delle strategie di reclutamento di questi gruppi e che spesso diventano, essi stessi, vittime di questa violenza estremista.

\subsection{Estremismo, radicalizzazione e terrorismo: una precisazione terminologica}

La terminologia che riguarda il fenomeno dell'estremismo violento è complessa e ancora in gran parte dibattuta. Ciò è dovuto a tutta una serie di ragioni, tra cui la principale è il fatto che molti termini utilizzati in questo campo non hanno delle definizioni universalmente accettate, motivo per cui si rende necessario, in questo contesto, precisare il significato di alcuni concetti chiave utilizzati in questo articolo.

Innanzitutto il concetto di "estremismo", che letteralmente significa "credere in e sostenere idee che sono molto lontane da ciò che la gran parte delle persone considera corretto o ragionevole» (UNESCO, 2019, passim). "Estremismo" fa quindi riferimento ad atteggiamenti o comportamenti che sono considerati fuori dalla norma. Nello stesso tempo, vi è una natura intrinsecamente soggettiva del termine, che può assumere diversi significati a seconda di chi definisce la norma e decide di conseguenza cosa è accettabile e cosa non lo è.

Mentre, sebbene per "estremismo violento" non esista una definizione concordata a livello internazionale, il significato comunemente attribuito a questa espressione e utilizzato dall'UNESCO (2019), è che si riferisca a convinzioni e azioni di persone favorevoli alla violenza o che vi ricorrono per raggiungere obiettivi ideologici, religiosi o politici. Fra questi si trovano il terrorismo e altre forme di violenza settaria e politicamente motivata. Il fulcro concettuale dell'estremismo violento è il ricorso all'uso della violenza per motivazioni ideologiche, normalmente basate su teorie cospirative.

Il termine "terrorismo" si riferisce, invece, a una particolare strategia adottata per conseguire un obiettivo politico, cioè la creazione e lo sfruttamento deliberato della paura. Le espressioni "estremismo violento" e "terrorismo" sono spesso erroneamente utilizzate come fossero sino- 
nimi. Tuttavia, mentre il terrorismo è una forma di estremismo violento ed è spesso motivato ideologicamente, la base concettuale del terrorismo che lo differenzia dall'estremismo violento è la creazione della paura o del terrore come mezzo per raggiungere un dato scopo.

McCauley e Moskalenko (2017) pongono la differenza tra opinioni radicali e azioni radicali, una differenza che talvolta viene indicata con le espressioni "radicalizzazione" intesa come un fenomeno per cui dei soggetti acquisiscono un'opinione radicale e "radicalizzazione violenta" che si manifesta con azioni violente. Sebbene l'estremismo violento venga inserito in una cornice dominata dalla teoria della scelta razionale, sta emergendo una maggiore consapevolezza sull'importanza delle emozioni e dell'esperienza affettiva. Emozioni come rabbia, oltraggio, vergogna, umiliazione e forme estreme di odio-amore possono spingere alla radicalizzazione delle idee o dell'azione ed il messaggio estremista viene fornito in modo da provocare reazioni emotive.

Nell'ambito degli sforzi volti a prevenire l'estremismo violento, il termine "radicalizzazione" è normalmente utilizzato per descrivere i processi attraverso i quali una persona adotta delle convinzioni o delle pratiche estreme rispetto a quelle nel corpo politico di riferimento in un dato momento storico al punto da legittimare l'uso della violenza. Il concetto-chiave in questo caso è proprio il processo che porta ad abbracciare la violenza. La radicalizzazione è dunque un processo durante il quale un individuo vive trasformazioni cognitive, che possono portare alla legittimazione della violenza e a un suo possibile utilizzo per raggiungere obiettivi politici. Le ragioni alla base del processo possono essere politiche, religiose, ideologiche, sociali, economiche o personali (Caparesi, 2019).

Le cause che spingono un/a giovane a radicalizzarsi sono molteplici e complesse, e ogni storia è un caso a sé, ma vi sono dei motivi di spinta (push factors) e di attrazione (pull factors). Tra i fattori principali di spinta ci sarebbero sentimenti di risentimento (per cause economiche, sociali e politiche, o senso di giustizia e discriminazione subito), crisi personali (crisi identitarie, lutti, eventi tragici), frustrazioni (esclusione sociale, essere vittima di bullismo o cyberbullismo, marginalizzazione, polarizzazioni) e caratteristiche personali come il fascino per la violenza, legami personali con i terroristi e disturbi mentali. Tra i fattori di attrattiva invece, si riscontrano: il senso di un obbligo morale (difendere la razza, il proprio gruppo, la propria zona o quartiere, senso di lealtà e impegno alla causa/ideologia o network), senso di appartenenza ad un gruppo e condivisione di norme, principi e stili di comportamento, desiderio di potere, desiderio di eccitamento e di avventura. Le tecniche di reclutamento sfruttano proprio le spinte motivazionali e i bisogni individuali, focalizzandosi sulla persuasione e sulla manipolazione emotiva. Naturalmente questo ha maggiormente effetto se si colpiscono soggetti fragili, in una posizione di dipendenza e subordinazione da qualcuno.

Il ruolo della Rete oggi assume una dimensione preponderante e, in Rete, l'estremismo politico e religioso assume varie forme. Tutti i discorsi estremisti hanno un punto in comune, ossia sono diametralmente opposti ai principi democratici della nostra società. Il mondo viene suddiviso in "amici e nemici" e i risentimenti nei confronti degli altri vengono fomentati (Pasta, 2019).

L'estremismo violento è divenuto una seria minaccia per le società di tutto il Mondo. Esso incide sulla sicurezza, sul benessere e la dignità di molte persone che vivono in Paesi sia sviluppati che in via di sviluppo e sui loro modi di vivere sostenibili e pacifici. L'estremismo violento rappresenta anche una grave sfida ai diritti umani. Ad oggi, le minacce poste dall'estremismo violento sono state valutate soprattutto attraverso la lente militare e della sicurezza (UNESCO, 2019). Dal documento UNESCO Prevenire l'estremismo violento attraverso l'educazione (2019) emerge che i governi sono sempre più convinti che rafforzare soltanto le misure di sicurezza sia insufficiente per proteggere i cittadini dagli attacchi terroristici commessi da estremisti violenti, 
se questo non si accompagna a un lavoro educativo di prevenzione primaria. Gli sforzi volti a prevenire l'estremismo violento devono essere considerati attraverso un approccio olistico, in particolare valorizzando l'educazione alla cittadinanza globale e alla resilienza durante tutto l'arco della formazione, dall'infanzia all'università.

In questo contesto, la quinta revisione della Strategia Globale delle Nazioni Unite contro il Terrorismo (30 giugno-1 luglio 2016) è stata un'occasione per sottolineare ancora una volta l'importanza di includere la prevenzione fra gli obiettivi della strategia e per accogliere con interesse il Piano d'Azione del Segretario Generale dell'ONU per la Prevenzione dell'Estremismo Violento (2015). Sempre in questa occasione, l'Assemblea Generale delle Nazioni Unite ha fatto appello agli Stati Membri perché, pur tenendo conto dei loro contesti nazionali, introducano le sue raccomandazioni con il sostegno delle Nazioni Unite. Fra le azioni prioritarie, si rileva la necessità di sostenere l'educazione, lo sviluppo e la facilitazione dell'occupazione come mezzi con cui promuovere il rispetto della diversità fra esseri umani e preparare i giovani ad accedere al mercato del lavoro.

Gli sforzi dell'UNESCO in questo campo contribuiscono alla realizzazione dell'Agenda Globale sull'Educazione 2030 e in particolare all'attuazione del traguardo 4.7 dell'Obiettivo di Sviluppo Sostenibile 4 (OSS 4) sull'educazione di qualità.

Nel 2011 la Commissione Europea ha lanciato la "Rete di sensibilizzazione al problema della radicalizzazione" ("Radicalisation Awareness Network" - RAN) con la premessa che il contrasto al terrorismo e all'estremismo violento non fosse solo un problema securitario, ma soprattutto un'attività preventiva necessaria per convincere i giovani a interrompere il loro coinvolgimento con gruppi violenti o convincerli ad allontanarsi dalle idee estremiste. La rete RAN coinvolge operatori che lavorano a diretto contatto con soggetti vulnerabili e provenienti da organizzazioni pubbliche e private. Viene riconosciuto un ruolo importante alla scuola e agli insegnanti nella prevenzione e nella fase iniziale di individuazione dei primi segnali d'allarme:

Gli insegnanti e gli educatori giocano un ruolo chiave nella prevenzione della radicalizzazione che conduce all'estremismo violento. Si confrontano con le idee estremiste, gli argomenti sensibili ed i comportamenti dei propri studenti. Qualunque siano le fonti di queste espressioni (percezione di ingiustizia, torti o pressioni dei pari) l'insegnante si trova nella difficoltà di identificare e trattare questi argomenti in modo aperto e sicuro. Lasciare queste espressioni senza risposta potrebbe alimentare la narrativa estremista e mettere a repentaglio la sicurezza degli studenti. C'è bisogno, pertanto, che gli insegnanti investano e ricevano sostegno aumentando la propria abilità nell'affrontare queste sfide e sapere come agire in questi casi ${ }^{3}$.

La formazione dei docenti in ottica interculturale rappresenta un pilastro fondamentale per aiutare gli studenti a sperimentare nella scuola pratiche di cittadinanza democratica che dovrebbero poi essere attuate nella società (Biagioli, 2020). Come scrive Biagioli, «il docente costruisce un curricolo pluridimensionale dove accanto e dentro le discipline trovano spazio dimensioni altre: quella delle competenze di cittadinanza e della transdisciplinarietà, dove le relazioni vengono concepite all'interno di un sistema totale, senza frontiere stabili tra le discipline» (2020, p. 20).

Quindi, nell'ottica di prevenzione primaria, la scuola gioca un ruolo basilare, ma, accanto ad essa, deve esserci il supporto e la collaborazione della famiglia, l'altra agenzia educativa deputata per eccellenza all'educazione informale, dove quotidianamente ragazzi e ragazze vivono, interagiscono e si formano.

${ }^{3}$ Documento pubblicato online all'indirizzo: https:/lec.europa.eu/home-affair/sites/homeaffairs/files/what-we-do/ networks/radicalisation_awareness_network/docs/manifesto-for-education-empowering-educators-and-schools_en.pdf, e https://euroclio.eu/wp-content/uploads/2016/03/Manifesto-for-Education-Empowering-educators-and-schools.pdf (data di ultima consultazione: 15.02.21); traduzione italiana a cura dell'Autrice, N.d.R. 


\section{Genere, estremismi e processi di radicalizzazione}

Il tema del terrorismo e, più in generale dei comportamenti estremisti e di radicalizzazione, fino ad oggi è stato scarsamente indagato dalla ricerca pedagogica (Tramma et al., 2019), ancora più trascurata risulta la dimensione di genere (Carter, 2013). Alla figura del terrorista è molto spesso, in maniera stereotipata, associato un uomo, immigrato, per lo più musulmano e questo rafforza tutta una serie di stereotipi e pregiudizi sedimentati nel tempo e rafforzati e diffusi dopo i tragici attentati terroristici a New York del 2001.

$\mathrm{Vi}$ è un rischio molto alto, sempre più diffuso, che le differenze culturali, linguistiche, etniche e religiose, collegate alle migrazioni internazionali, tendano a polarizzarsi in una dinamica di causa-effetto con il terrorismo (Brambilla, 2019), alimentando così un circolo vizioso di discriminazioni, forme di razzismo culturale e attivatore di violenza simbolica ed agita. Gli studi di genere, in questo senso, stanno riuscendo ad offrire una lettura in ottica di genere dei processi e atti terroristici che rivelano ancora una volta, i meccanismi di subordinazione delle donne al potere maschile.

Sebbene vi siano ancora poche ricerche sul rapporto tra differenza di genere e processi di radicalizzazione, quelle attualmente esistenti stanno evidenziando la complessità e la poliedricità del problema, le discriminazioni e gli stereotipi di genere sia nei processi di reclutamento delle vittime sia nei processi di narrazione e attribuzione di significato degli episodi terroristici (Cruise, 2016). L'attenzione alla dimensione di genere in questo tipo di ricerche, ha permesso di indagare le strutture e le dinamiche di potere e di disuguaglianza sottese alle dinamiche terroristiche, nell'arruolamento, nelle narrazioni e nelle pratiche antiterroristiche.

Secondo una prospettiva di genere, la radicalizzazione delle donne e il loro coinvolgimento in gruppi estremisti è sottostimato ed è diffusa l'idea che il terrorismo riguardi esclusivamente gli uomini (EPRS, 2018). Recenti ricerche rivelano che il 17\% dei Foreign fathers europei sono rappresentati da donne e, nel 2016, su quattro persone arrestate in Europa per attività terroristiche, una è donna. Inoltre, negli ultimi anni vi è stato un rapido incremento della presenza femminile nelle attività terroristiche: nel 2016, tra le persone arrestate in Europa per attività terroristiche, il 26\% era rappresentato da donne rispetto al 18\% del 2015 (EPRS, 2017).

Leggere i processi di radicalizzazione in un'ottica di genere, significa da un lato indagare e riconoscere la potenziale minaccia derivante dalle donne radicalizzate, dall'altro, occorre anche prestare maggiore attenzione alla dimensione di genere delle strategie di de-radicalizzazione e al contributo specifico che le donne possono dare per la prevenzione di fenomeni di questo tipo.

L'Organizzazione per la Sicurezza e la Cooperazione in Europa (OSCE) riporta che il potenziale di radicalizzazione e coinvolgimento delle donne in gruppi estremisti violenti continua ad essere relativamente sottovalutato, poiché è diffusa la concezione che l'estremismo violento e il terrorismo riguardino quasi esclusivamente gli uomini. Tuttavia, la ricerca dimostra che, storicamente, le donne sono state attive in organizzazioni politicamente violente in diverse regioni del mondo, non solo come ausiliarie ma anche come leader nell'organizzazione, nel reclutamento e nella raccolta di fondi e in ruoli operativi diretti. L'uso crescente di internet sta permettendo sempre più alle donne di unirsi alle organizzazioni radicali che divengono così accessibili virtualmente e anonimamente (EPRS, 2018).

Le donne si uniscono ai movimenti terroristici per molti degli stessi gruppi di ragioni psicologiche, personali, sociali, economiche e politiche che hanno gli uomini, oltre a un maggiore senso di ribellione e riscatto per la loro posizione di dipendenza e subordinazione che in quanto donne sono costrette a subire, in particolare in alcune culture (EPRS, 2018; Sikkens et al, 2017).

Tra i fattori scatenanti che spingerebbero le donne ad arruolarsi in organizzazioni terroristiche emergono: ribellione e desiderio di azione; una spinta al potere e la promessa di avventura; 
un'attrazione per la politica; e l'impegno per una causa specifica, ideologia religiosa. Il Centre on Global Counter terrorism Cooperation (CGCC) aggiunge una serie di ulteriori fattori di spinta per entrambi i sessi, tra cui insoddisfazione per le condizioni socio-politiche, il dolore per la morte di una persona cara, l'intenzione di trarre benefici economici o il desiderio di innescare un cambiamento radicale della società.

Tuttavia, si evidenzia una maggiore fragilità delle donne proprio dovuta a stereotipi e discriminazioni legate all'appartenenza al genere femminile che le rende maggiormente vulnerabili ed a rischio di cadere vittime di organizzazioni radicali e terroristiche.

A livello generale, come ha sottolineato l'OSCE (2018), fattori come la disuguaglianza e la discriminazione basate sul genere, la violenza contro le donne, bassi livelli di istruzione, povertà economica ed educativa contribuiscono ad incrementare la discriminazione, inoltre, la violenza subita e la mancanza di opportunità, possono agire come fattori specifici nella radicalizzazione delle donne.

L'esperienza di vivere in una società che nega loro pieni diritti civili e opportunità economiche può portare alcune donne a percepire la partecipazione al terrorismo come un modo per acquisire libertà, emancipazione, rispetto e uguaglianza. D'altra parte, le violazioni dei diritti umani delle donne possono anche acutizzare i loro sentimenti di alienazione, isolamento ed esclusione che possono renderle individui più suscettibili alla radicalizzazione. Anche il trauma personale può essere una delle motivazioni fondamentali per il coinvolgimento delle donne nell'estremismo violento, ad esempio l'esperienza dello stupro sembrerebbe un fattore di motivazione delle donne a diventare attentatrici suicide (OSCE, 2018).

In alcune comunità le donne vittime della violenza sessuale, sono ritenute anche responsabili di questo e quindi accusate di portare vergogna alle loro famiglie: diventare un'attentatrice suicida può essere un modo per rimuovere la vergogna dello stupro e sostituirla con l'orgoglio associato all'essere una "martire per la causa".

Anche i tradizionali ruoli di genere possono influenzare la radicalizzazione delle donne. Per esempio, è stato notato che relazioni di parentela (essere figlie o sorelle di un terrorista) e di amore (mogli) possono giocare un ruolo importante nell'attirare le donne in organizzazioni estremiste e terroristiche. Talvolta la presenza di una donna in un'organizzazione terroristica è dovuta all'essere stata legata come moglie, madre o figlia ad un uomo di cui sente il dovere di vendicare la morte, come accade per le «spose della jihad» (Cunningham, 2007, passim)

La propaganda usata per reclutare le donne contrappone narrazioni negative dell'esperienza delle donne musulmane nelle società occidentali con narrazioni positive sul loro contributo al nuovo "stato". Le ragioni per cui le donne viaggiano per unirsi all'ISIL/Dảesh sono diverse, e comprendono un'ampia gamma di fattori di spinta e di attrazione. Nei paesi a maggioranza musulmana da cui proviene la maggior parte delle reclute dell'ISIL/Da’esh, le donne trovano nella nuova organizzazione terroristica, una forma di libertà dalle tradizioni patriarcali e una fuga dalle restrizioni. Fare parte di un'organizzazione e avere un proprio ruolo all'interno di essa rappresenterebbe in qualche modo una specie di riscatto sociale e una specie di spazio per esercitare la propria libertà (Sikkens et al., 2017).

Emerge quindi una doppia discriminazione e vittimizzazione femminile: da una parte, esiste una cultura che impedisce alle donne la libertà di essere e di realizzarsi e dall'altra, il rischio di cadere in un sistema terroristico che decide il loro ultimo destino. Le donne che sono coinvolte in atti violenti sono spesso rappresentate come l'eccezione poiché le donne sono ritenute intrinsecamente pacifiche e meno inclini alla violenza per loro natura rispetto agli uomini e questa percezione contribuisce alla sedimentazione di uno stereotipo di genere.

Le donne possono svolgere ruoli multipli nelle organizzazioni terroristiche e questo avviene soprattutto sfruttando gli stereotipi di genere precedentemente citati che ritengono le donne 
poco inclini alla violenza. Le organizzazioni stanno sempre più sfruttando questi stereotipi usando le donne per superare il personale di sicurezza, evitare il rilevamento ed aumentare l'attenzione dei media (Carter, 2013; EPSR, 2018). Nel frattempo, le donne stanno sempre più assumendo ruoli attivi nelle operazioni terroristiche, in particolare negli attentati suicidi. Gli esperti dichiarano che le donne attentatrici hanno commesso più di 257 attacchi suicidi tra il 1985 e il 2010 (che rappresentano circa un quarto del totale) per conto di diverse organizzazioni terroristiche (EPSR, 2018). Dove le donne non sono in tali posizioni, possono essere simpatizzanti dei terroristi, sostenitrici di ideologie radicali e mobilitatrici. Vengono "sfruttati" anche i ruoli tradizionali delle donne come mogli, madri e nutrici, che le autorizzano a diventare protettrici dei valori culturali, sociali e religiosi e di trasmetterli alla generazione successiva, in questo modo possono mantenere le organizzazioni terroristiche attraverso la loro propaganda, il reclutamento, la raccolta di fondi e altre attività di supporto.

La responsabilità femminile è stata ritratta, prima di tutto, come essere una buona moglie (sposa jihadista) per il marito jihadista, e diventare una madre per la prossima generazione del jihadismo.

Per quanto concerne il ruolo della famiglia originaria, in particolare dei genitori, nei processi di radicalizzazione dei giovani, uno studio condotto recentemente (Sikkens et al., 2017) dimostra che la famiglia, in particolare nel caso di famiglie problematiche, rappresenta un fattore di rischio verso la radicalizzazione mentre la famiglia "funzionale" e supportiva può avere un importante ruolo facilitante nei processi di integrazione sociale. Questo aspetto è molto importante per incentivare politiche di supporto e di sostegno alla genitorialità.

\section{Discriminazioni e rischi tra le giovani e le donne musulmane in Occidente. Esiti di una ricerca condotta nella scuola secondaria}

Le donne immigrate, proprio perché donne e migranti, riuniscono una doppia specificità di disconoscimento di identità e di espropriazione culturale (Mussi, 2019; Ulivieri, 2017). Le discriminazioni legate al genere e alla provenienza etnica si incontrano e si interconnettono rendendo ancora più difficile la loro socializzazione e la loro integrazione nella società. L'appartenenza al genere femminile e la provenienza da una cultura altra inducono verso le donne una doppia stigmatizzazione, dando origine a percorsi esistenziali complessi e difficili, spesso in conflitto tra il mantenimento della cultura originaria e l'influenza della cultura accogliente (Bolognesi, 2017, 2018; D’Ignazi, 2008).

In particolare, le adolescenti e le giovani incarnano proprio l'anello di congiunzione tra due culture e il passaggio e la trasformazione della cultura familiare (Guerrini, 2018). Negli ultimi anni, una particolare attenzione da parte della ricerca pedagogica è stata dedicata alle giovani ragazze e donne musulmane (Acocella, Peppicelli, 2015; Biagioli, 2017; Salih, 2008), sia perché rappresentano una parte considerevolmente importante da un punto di vista quantitativo della popolazione, sia perché per il fatto di indossare il velo le rende maggiormente vittime di stereotipi e discriminazioni tra i quali, pesa in maniera significativa la paura del terrorismo (Allevi, 2009, 2017; Jelloun, 2017).

In Italia, attualmente vi sono circa 300mila studenti provenienti da popolazione di cultura islamica (prevalentemente Marocco, Tunisia, Egitto, Algeria). Tra gli studenti stranieri, quelli musulmani sfiorano il 38\% del totale e rappresentano il 3,3\% della popolazione studentesca, sono concentrati soprattutto nelle scuole del Nord e Centro Italia poiché vi è una maggiore concentrazione di industrie e possibilità di lavoro nel terzo settore per i loro genitori (Fiorucci, Pinto Minerva et. al., 2017). 
Le ragazze nate in Italia, o arrivate da piccole e cresciute qui sono l'immagine di una fase difficile di transizione, circondate dalla diffidenza verso un mondo islamico percepito come nemico dell'Occidente (Santerini, 2017). L'Islam può essere uno dei riferimenti identitari anche se non l'unico e soprattutto variabile e mutabile a seconda delle esperienze di vita della ragazza stessa, dei modelli educativi familiari e scolastici (Acocella, Peppicelli, 2015).

Durante una ricerca condotta nel 2017 tra studenti e studentesse di alcune scuole secondarie di secondo grado toscane, attraverso delle interviste semi-strutturate, sono state raccolte testimonianze molto significative a questo proposito, di cui si riportano alcuni passít:

Sono musulmana e voglio continuare ad essere musulmana ma non rigida come mio padre, lui è troppo severo... Per lui dovrei frequentare solo persone musulmane. Ogni giorno che passa mi sento più italianizzata e mi faccio sempre più domande su quali siano i comportamenti da evitare... (Ragazza egiziana, 18 anni).

Qualche volta vengo presa in giro perché sono musulmana e porto il velo, ma non a scuola, questo avviene soprattutto fuori, per la strada, quando sono in centro (Ragazza, marocchina, 17 anni).

Dai dati raccolti in questa ricerca è emerso che la discriminazione di genere qualche volta si intreccia con la discriminazione etnica-culturale, soprattutto quando le differenze sono ben visibili, come nella religione islamica, e richiedono pratiche religiose diverse dalla cultura occidentale. In questo senso, essere donna musulmana è diventato un elemento di forte rischio di esclusione/emarginazione dalle dinamiche relazionali adolescenziali.

Spesso i giovani islamici vanno incontro a una serie di pregiudizi da parte della comunità aggravati da due fattori: l'ignoranza verso la cultura islamica e sulle differenze nazionali e la propaganda mediatica nei confronti della religione islamica (Allevi, a cura di, 2009; Id., Guolo, Rhazzali, a cura di, 2017). Il velo cattura subito l'attenzione, ci trasmette delle informazioni e un certo modo di vivere della donna. Durante un focus group, un ragazzo ha affermato:

Io credo che tra uomini e donne non ci sia una completa parità, le donne sono considerate inferiori perché meno adatte a comandare e questo non va bene, è uno stereotipo. Ma io credo che agli uomini non piaccia essere comandati da una donna. Se poi una donna è straniera, ancora peggio potrebbe avere ancora più problemi, non capire la lingua... Un giorno, mentre ero in un negozio del centro ho sentito una commessa dire che non potevano assumere una donna musulmana perché porta il velo e veste in un certo modo e nel negozio non sta bene. Poi io credo che un motivo sia che le persone hanno paura del terrorismo, hanno paura che le donne sotto ai vestiti larghi possano nascondere un'arma o delle bombe (Ragazzo italiano, 14 anni).

Da un'ulteriore ricerca condotta in Italia tra il 2015 e il 2017 tra ragazze (14-19 anni) nate o cresciute in Italia in famiglie migranti, è emerso come il collegamento tra Islam e terrorismo può tradursi in micro azioni quotidiane di discriminazione ad opera di conoscenti e non, oltre alla richiesta di distanziamento dalla propria fede religiosa. Inoltre, tale situazione si aggrava nei giorni immediatamente successivi agli attentati jihadisti e viene denunciata dalle giovani come un elemento di disagio e disturbo al loro processo di crescita e di inclusione sociale (Pozzebon, 2019).

${ }^{4}$ Si tratta della Ricerca Strategica di Ateneo "Intercultura e ruolo delle istituzioni educative per la crescita umana. Dal modello compensativo alla scuola inclusiva" 2014-2017, presso il Dipartimento di Scienze della Formazione e Psicologia - oggi FORLILPSI - dell'Università degli Studi di Firenze, coordinato dalla Prof.ssa Simonetta Ulivieri, N.d.A. 
I ragazzi vissuti nella società italiana si troverebbero a vivere in una condizione di doppia etnicità che li porterebbe a sentirsi costantemente in bilico tra le due culture. Una delle tendenze più pericolose per gli autoctoni è quella di pensare alle culture altre come essenze omogenee, predefinite e contrapposte le une alle altre. Queste percezioni hanno prodotto notevoli implicazioni anche sul piano delle rappresentazioni di genere (Tarabusi, 2010), ad esempio, le donne migranti sono state considerate spesso come portatrici e riproduttrici delle tradizioni locali, depositarie dei modelli culturali e dei valori della cultura di origine. Questa visione etnocentrica/androcentrica ha contribuito a diffondere nelle società di accoglienza un'immagine stereotipata delle donne migranti, viste come vittime passive, in posizione di subalternità e in contrapposizione alle donne occidentali orientate all'emancipazione e al progresso.

Dalle interviste con le insegnanti, sono emerse alcune testimonianze che rivelano delle difficoltà, talvolta quasi un sentimento di timore e scoraggiamento da parte delle insegnanti stesse per difficoltà derivanti dai tentativi di convivenza e conciliazione delle due culture, quella italiana e quella islamica.

Noto che spesso le madri musulmane hanno paura a parlare, anche con noi insegnanti, sono escluse dalla vita pubblica, dovrebbe essere fatto qualcosa per coinvolgerle e farle partecipare attivamente. A scuola non vediamo mai mamme musulmane a parlare con i docenti, solo padri. Le figlie sono molto educate e riservate $\mathrm{e}$ meno coinvolte attivamente nella vita scolastica. Occorre che la scuola si apra e promuova occasioni di incontro, la nostra scuola, anche se tra tante difficoltà, sta iniziando a farlo (Docente di lingua italiana).

Quando al terzo anno i ragazzi e le ragazze scelgono la scuola superiore, mi rendo conto che per qualche ragazza musulmana, più tradizionalista, è come se la scelta fosse già stata fatta, ossia di lasciare gli studi e

dedicarsi alla famiglia e alla casa. È un peccato perché qualcuna di loro avrebbe delle eccellenti capacità ma deve interrompere gli studi per dedicarsi alla famiglia. La scuola deve lavorare molto per il loro orientamento (Docente di matematica e scienze).

Le parole delle due insegnanti indicano la pista da seguire: lavorare sull'inclusione speciale delle madri, sul rafforzamento del loro senso di empowerment e autostima affinché possano esercitare un ruolo partecipativo nella comunità scolastica e familiare e contribuire ancora più attivamente al processo di crescita cognitiva, psicologia e sociale dei figli e delle figlie.

Le giovani donne islamiche in Italia hanno il delicato e complesso compito di "traduzione" dei valori familiari (in costante mutamento) a quelli ancora più variegati e frammentati della cultura di accoglienza. A loro spetta l'importante ruolo di mediazione continua tra culture e di interpretare in modo attivo questi cambiamenti (Granata, 2011) che può renderle protagoniste nello loro processo di integrazione sociale e di facilitazione in quello dei familiari ma può esporle anche al rischio di fallimenti, chiusura sociale, sentirsi vittima di discriminazione con tutte le conseguenze che questo può portare.

Per le donne, spesso madri, è più difficile essere pronte ad accogliere i nuovi stimoli culturali e apprendere la lingua, rimanendo in una situazione di interno/esterno alla comunità (Bolognesi, 2017). Infatti, sono le madri che si occupano della cura, della crescita e dell'educazione dei figli, ma sia per difficoltà linguistiche, sia talvolta per motivi culturali che le spingono a stare ai margini e non partecipare attivamente alla vita comunitaria, spesso non partecipano alla vita scolastica dei figli e delle figlie.

\section{Da vittime a costruttrici di comunità: strategie di rafforzamento della coesione sociale attraverso il Progetto europeo "CommUnity"}

Fino a quanto enunciato fino ad adesso, le ricerche e la letteratura scientifica sembrerebbero dimostrare un maggiore vulnerabilità delle donne e delle giovani immigrate, rischio di emar- 
ginazione sociale, subordinazione e anche di cadere in comportamenti estremisti e terroristici. Dall'altra parte, però le donne, ed in particolare le madri, hanno una grande opportunità da un punto di vista educativo: quella di poter svolgere un ruolo fondamentale nell'educazione dei figli e quindi poter intercettare eventuali segnali d'allarme, facilitare l'ingresso dei figli e delle figlie nel mondo sociale, contribuire al mantenimento della cultura originaria.

La famiglia immigrata, come quella autoctona, è rappresentata da una pluralità di modelli. Una pluralità, ancora più variegata, poiché arricchita da fattori connessi alla diversità etnica, linguistica, culturale, religiosa e dal particolare vissuto dei migranti. Ogni famiglia, immigrata o non, costituisce un nucleo a sé, un microcosmo tenuto insieme da legami e da una storia, contrassegnato da ruoli, risorse, affetti ed eventi (Silva, 2009, p. 30).

Educare i figli, per le famiglie immigrate, è indubbiamente un compito tra i più difficili, per diverse ragioni che cerchiamo qui di sintetizzare. Si tratta in primo luogo di offrire ai propri figli riferimenti culturali e valori che permettano loro di transitare tra più culture, di poter appartenere allo stesso tempo a più paesi, di costruire un'identità capace di conciliare i valori della tradizione familiare e quelli del Paese dove sono nati e/o cresciuti (Ibidem).

L'educazione familiare dovrebbe tener conto delle differenti aspettative sociali delle nuove generazioni per aiutare i figli ad affrontare le difficoltà che hanno di fronte. Per poter essere una doppia risorsa, sia per la società d'arrivo sia per sé medesima, la famiglia immigrata va però sostenuta non solo materialmente, ma anche attraverso percorsi di educazione familiare e di sostegno alla genitorialità. La vulnerabilità femminile deve essere trasformata in elemento di forza per le famiglie e per la società. Il coinvolgimento delle donne è essenziale per affrontare e prevenire efficacemente il terrorismo poiché le donne rappresentano "la prima linea di protezione" nella prevenzione dell'estremismo (OCSE, 2013).

Dalle prime forme di educazione familiare i giovani si abituano a un atteggiamento costruttivo di apertura e dialogo verso l'altro. La famiglia può ancora giocare un ruolo importante nella protezione degli studenti a rischio. Perché questo accada, i genitori devono comprendere i processi di radicalizzazione che possono portare alla violenza e devono essere aiutati a sviluppare le giuste competenze per svolgere un ruolo attivo nella promozione di atteggiamenti positivi verso la non violenza. Infatti, le madri e i padri possono effettivamente esercitare un'influenza particolarmente costruttiva se sanno come comunicare con i loro figli in modo utile, non solo facendo la morale (UNESCO, 2016, p. 6).

In particolare, la maternità e il ruolo delle donne come educatrici possono conferire loro un potere speciale nella lotta contro il terrorismo e l'estremismo (Women's Rights Committee of the Union for the Mediterranean, 2016). Occorre mettere le donne in condizioni di trasformare la loro condizione di dipendenza e mancanza di senso e di obiettivi in una situazione di soddisfazione e stabilità per costruire progetti, avere degli obiettivi, un ruolo sociale e un lavoro.

Il Progetto europeo "CommUnity" ${ }^{\text {, }}$ di cui il Dipartimento di Formazione, Lingue, Intercultura, Letterature e Psicologia dell'Università degli Studi di Firenze è partner, sotto la supervisione scientifica di Raffaella Biagioli, ha come obiettivi prioritari la prevenzione e l'eliminazione della radicalizzazione violenta nelle società europee attraverso il coinvolgimento di vari attori locali e il rafforzamento della coesione sociale. Per raggiungere tali obiettivi e soprattutto per

${ }^{5}$ Laddove non diversamente specificato, d'ora in avanti le traduzioni italiane dei documenti UNESCO citate sono reperibili sul sito dell'Organizzazione; per tutti i dettagli si rimanda ai Riferimenti bibliografici e sitografici, N.d.R.

${ }^{6}$ Il sito del Progetto è il seguente: www.thecommunityproject.eu (data di ultima consultazione: 10.11.21), N.d.A. 
incrementare il senso di appartenenza dei giovani è previsto l'utilizzo di varie tipologie di forme artistiche, con un'attenzione particolare verso le comunità musulmane e il ruolo delle donne.

Alla base vi è la concezione dell'arte intesa come linguaggio universale e che quindi attraverso le varie forme di espressione possa aiutare a sconfiggere i pregiudizi, la paura del diverso e le ostilità (Borrup, 2006). Quindi, all'interno del Progetto, sono stati realizzati cineforum nelle scuole secondarie e nell'università per abituare i giovani a riflettere su tematiche quali il dialogo interculturale e interreligioso, attraverso cineforum e dibattiti, caffè letterari, mostre di pittura e fotografia, festival artistici.

Particolare attenzione è stata posta sulle donne musulmane per renderle protagoniste dei loro processi di empowerment e per metterle sempre più in interazione con la comunità accogliente. Per fare questo occorre che si realizzi una produttiva ed efficace collaborazione e corresponsabilità tra scuola e famiglia e che il personale scolastico-educativo sia adeguatamente formato. Per facilitare il processo di integrazione delle famiglie non italiane, è importante che la scuola eserciti un ruolo facilitante nell'interazione tra famiglie italiane e non, iniziando a lavorare sugli aspetti in comune e sulle differenze, come ad esempio il fatto che tutti i genitori sono genitori di figli adolescenti e considerando la genitorialità come condizione umana trasversale alle varie culture (Cestaro, 2017).

La famiglia è il contesto all'interno del quale prende avvio la "dinamica appartenenza identità" come serie di vincoli ma anche di possibilità di affermare sé stessi. Come scrive Mussi (2017), i genitori migranti hanno la possibilità di offrire ai propri figli i riferimenti culturali e valoriali che possano permettere loro di transitare tra più culture e tale dimensione deve essere supportata da programmi di sostegno alla genitorialità.

Tali programmi dovrebbero essere basati sulla pratica della riflessività che permetta ai genitori di riflettere sui propri modelli agiti di genitorialità, sui valori trasmessi ai figli e alle figlie e dovrebbe accogliere tutti i dubbi e le perplessità che tali dinamiche possono scatenerà.

In questi processi, sarà necessario assumere un atteggiamento di decentramento culturale che prevede «l'assunzione di uno sguardo sull'altro senza ricondurlo a categorie proprie di riferimento" (Mussi, 2017, p. 259). Il dialogo tra insegnanti (operatori, educatrici) e famiglie immigrate può diventare così un'occasione di vero dialogo: una creazione di nuovi significati, continuamente condivisi e rinegoziati.

\section{Conclusioni}

L'indagine sui comportamenti estremisti dei giovani e delle giovani, soprattutto nell'ottica della dimensione di genere ha aperto molte piste di ricerca che necessitano di essere affrontate dalla ricerca pedagogica. Innanzitutto, è necessario incrementare la consapevolezza della radicalizzazione del terrorismo femminile e del coinvolgimento delle donne nel terrorismo, incoraggiando la sensibilizzazione di genitori, insegnanti, assistenti sociali, agenti di polizia in prima linea, giornalisti e giudici per dissipare stereotipi e idee sbagliate.

Ancora una volta, l'educazione e, in particolare, la sinergia tra educazione familiare e educazione scolastica di buona qualità, possono aiutare a creare le condizioni che rendono più difficile la diffusione di ideologie estremiste violente. Le politiche educative possono far sì che $\mathrm{i}$ luoghi dell'apprendimento non diventino terreno fertile per l'attecchire dell'estremismo violento; inoltre, possono garantire che i contenuti della formazione e gli approcci di insegnamento e apprendimento sviluppino la resilienza dei discenti all'estremismo violento.

Il ruolo dell'educazione è, quindi, non tanto quello di intercettare estremisti violenti o identificare persone che potrebbero potenzialmente diventare dei terroristi, quanto creare le 
condizioni che permettano ai discenti di tutelarsi contro ogni forma di estremismo, rafforzando il loro impegno a favore della non-violenza e della pace.

In questo contributo l'attenzione si è maggiormente concentrata sulle forme di radicalizzazione e discriminazione legate al terrorismo di natura islamica, con la consapevolezza che si tratta di una lettura parziale e limitata del fenomeno poiché sono sempre più diffuse varie forme di estremismo, come gli estremismi delle forze di destra, che necessitano di approfondite piste di ricerca.

La dimensione di genere ha una forte influenza nei meccanismi di reclutamento e di prevenzione della radicalizzazione e soprattutto come dimostrano alcune ricerche, le condizioni di subordinazione di alcune donne le pongono a maggiore rischio di compiere scelte estremiste e di radicalizzazione. Per questo è necessario promuovere forme di empowerment femminile: a partire dalla scuola e dalla famiglia, nei confronti delle ragazze/donne straniere. Quindi, si rende sempre più necessaria e urgente un'adeguata formazione in ottica interculturale del corpo docente (Biagioli, 2018, 2020) e politiche educative di sostegno alla genitorialità (Silva, 2009).

Il ruolo delle famiglie immigrate e in particolare delle madri può divenire una risorsa strategica nel processo di inclusione sociale e rafforzamento della coesione sociale (Mussi, 2017). Affinché questo avvenga, occorre anche il supporto di politiche educative inclusive, di strategie comunicative basate sul dialogo, sulla riflessività e sulla co-costruzione di nuovi significati condivisi. da parte dei professionisti che lavorano nel settore educativo. nei processi educativi.

Dal punto di vista della ricerca pedagogica, occorre dare sempre più spazio alla sinergia tra gli studi di genere, la pedagogia di genere e la pedagogia interculturale (Lopez, 2018) per individuare precocemente i fattori di rischio verso gli estremismi e costruire percorsi educativi mirati all'inclusione sociale dei soggetti più vulnerabili.

\section{Riferimenti bibliografici}

Acocella I., Peppicelli R. (2015): Giovani musulmane in Italia. Percorsi biografici e pratiche quotidiane. Bologna: il Mulino.

Allevi S. (a cura di) (2009): Imusulmani e la società italiana. Percezioni reciproche, conflitti culturali, trasformazioni sociali. Milano: FrancoAngeli.

Allevi S., Guolo R. e Rhazzali M.K. (a cura di) (2017): Imusulmani nelle società europee. Appartenenze, interazioni, conflitti. Milano: Guerini e Associati.

Antonacci F., Gambacorti-Passerini M.B., Oggionni F. (a cura di) (2019): Educazione e terrorismo. Posizionamenti pedagogici. Milano: FrancoAngeli.

Barone P. (2009): Pedagogia dell'adolescenza. Milano: Guerini e Associati.

Barone P. (2011): Pedagogia della marginalità e della devianza. Milano: Guerini e Associati.

Bauman S. (2005): Vita liquida. Trad. it. Roma-Bari: Laterza, 2008.

Ben Jelloun T. (2016): Il terrorismo spiegato ai nostri figli. Trad. it. Milano: La Nave di Teseo, 2017.

Besozzi E. (a cura di) (2009): Tra sogni e realtà. Gli adolescenti e la transizione alla vita adulta, Roma: Carocci.

Biagioli R. (2017): Figlie di immigrati nella scuola italiana. Pedagogia Oggi, n. 1, pp. 205-222.

Biagioli R. (2018): Traiettorie migranti. Minori stranieri non accompagnati. Racconti e storie di vita. Pisa: ETS.

Biagioli R., Proli M.G., Gestri S. (2020): La ricerca pedagogica nei contesti scolastici multiculturali. Pisa: ETS.

Bolognesi I. (2017): Immigrant Mothers and Schools. Relations with Teachers. Pedagogia Oggi. n. 1, pp. 193-204.

Bolognesi I. (2018): Migrazioni e percorsi scolastici di adolescenti figli di genitori immigrati. In S. Ulivieri (a cura di): Ragazze e ragazzi stranieri a scuola. Pisa: ETS, pp. 193-204.

Borrup T. (2006): The Creative Community Builder's Handbook: How to Transform Communities Using Local Assets, Arts and Culture. Saint Paul, Minnesota: Turner Publishing Company.

Brambilla L., Galimberti A., Tramma S. (a cura di) (2019): Educazione e terrorismo. Milano: FrancoAngeli.

Brambilla L. (2019): Terrorismo, genere, donne. In Id., A. Galimberti, S. Tramma (a cura di) (2019): Educazione e terrorismo. Milano: FrancoAngeli, pp. 131-141. 
Cambi F. (2018): Introduzione a Mancaniello M.R., Per una pedagogia dell'adolescenza. Società complessa e paesaggi della metamorfosi identitaria. Lecce: Pensa Multimedia, pp. 9-12.

Caparesi C. (2019): Prevenzione dei radicalismi tra prospettive e buone prassi. In M. Bombardieri, M.C. Giorda, S. Hejazi. Capire l'Islam. Mito o realtà. Brescia: Morcelliana, pp. 265-294.

Carter B. (2013): Women and Violent Extremism (GSDRC Helpdesk Research Report 898). Birmingham (UK): GSDRC, University of Birmingham.

Cestaro M. Genitori di "seconda generazione": agenti di integrazione e di mediazione culturale nelle città. Formazione Lavoro Persona, n. 22, pp. 110-122.

Cruise R.S. (2016): Enought with the Stereotypes: Representations of Women in Terrorist Organizations. Social Science Quarterly, 97(1), pp. 33-43.

Cunningham K.J. (2007): Countering Female Terrorism. Studies in Conflict \& Terrorism, n. 30, pp. 113-129.

D'Ignazi P. (2008): Ragazzi Immigrati. L'esperienza scolastica degli adolescenti attraverso l'intervista biografica. Milano: FrancoAngeli.

Dusi P. (2017): Appartenenza, appartenenze. In M. Fiorucci, F. Pinto Minerva, A. Portera (a cura di): Gli alfabeti dellintercultura. Pisa: ETS, pp. 55-68.

European Parliament Research Service - EPRS (2018): Radicalisation and Counter Radicalisation: a Gender Perspective (https://www.europarl.europa.eu/EPRS/EPRS-Briefing-581955-Radicalisationgender-perspective-rev-FINAL.pdf; data di ultima consultazione: 26.02.21).

Erikson E. (1980): Infanzia e società. Trad. it. Roma: Armando Editore, 1980.

Fiorucci M., Catarci M. (2015): Il mondo a scuola. Per un'educazione interculturale. Roma: Edizioni Conoscenza.

Fiorucci M., Pinto Minerva F., Portera A. (a cura di) (2017): Gli alfabeti dell'intercultura. Pisa: ETS.

Giscard d'Estaing S. (2017): Engaging Women in Countering Violent Extremism: Avoiding Instrumentalisation and Furthering Agency. Gender \& Development, 25(1), pp. 103-118.

Granata A. (2011): Sono qui da una vita. Dialogo aperto con le seconde generazioni. Roma: Carocci.

Guerrini V. (2018): Le interviste agli adolescenti di seconda generazione a Firenze. Una riflessione pedagogica. In S. Ulivieri (a cura di): Ragazze e ragazzi stranieri a scuola. Pisa: ETS, pp. 245-270.

Guerrini V. (2019): Educare alla cittadinanza democratica al tempo del terrorismo globale. Il ruolo della scuola. In F. Antonacci, M.B. Gambacorti-Passerini, F. Oggionni (a cura di) (2019): Educazione e terrorismo. Posizionamenti pedagogici. Milano: FrancoAngeli, pp. 94-104.

Lopez A.G. (2018): Pedagogia delle differenze. Pisa: ETS.

Mancaniello M.R. (2002): L'adolescenza come catastrofe. Modelli d'interpretazione psicopedagogica. Pisa: ETS.

Mancaniello M.R. (2018): Per una pedagogia dell'adolescenza. Società complessa e paesaggi della metamorfosi identitaria. Lecce: Pensa Multimedia.

McCauley C., Moskalenko S. (2017): Understanding Political Radicalization: The Two-pyramids Model. American Psychologist, 72(3), pp. 205-216.

Mussi A. (2017): Integrazione a partire dalle madri. Discussione di due buone pratiche di supporto alla genitorialità migrante al femminile sul territorio milanese. Formazione Lavoro Persona, n. 22, pp. 257-266.

Mussi A. (2019): Islamofobia al femminile tra pregindizi e occasioni di dialogo. In L. Brambilla, A. Galimberti, S. Tramma (a cura di) (2019): Educazione e terrorismo. Milano: FrancoAngeli, pp. 154-166.

OSCE Secretariat OSCE ODIHR Expert Roundtables. (2013): Women and Terrorist Radicalization. Final report https://www.osce.org/atu/99919 (ultima consultazione: 20.02.2021)

Pasta S. (2019): Una lettura della Jahadosphera. Limportanza del web e dei legami deboli nell'educazione al terrorismo. In F. Antonacci, M.B. Gambacorti-Passerini, F. Oggionni (a cura di) (2019): Educazione e terrorismo. Posizionamenti pedagogici. Milano: FrancoAngeli, pp. 23-34.

Pozzebon G. (2019): Giovani donne musulmane in Italia e terrorismo: implicazioni pedagogiche e responsabilità educative. In L. Brambilla, A. Galimberti, S. Tramma (a cura di) (2019): Educazione e terrorismo. Milano: FrancoAngeli, pp. 142-153.

Salih R. (2008): Musulmane rivelate. Donne, Islam, modernità. Trad. it. Roma: Carocci.

Santerini M. (2017): Donne immigrate e nuova cittadinanza democratica. Pedagogia Oggi, n.1, pp. 25-38. 
Silva C. (2009): Famiglie immigrate e educazione dei figli. RIEF-Rivista Italiana di Educazione Familiare, n. 1 , pp. 30-36.

Silva C. (2015): Lo spazio dellintercultura. Milano: FrancoAngeli.

Silva C. (2017): Cittadinanza. In M. Fiorucci, F. Pinto Minerva, A. Portera (a cura di): Gli alfabeti dell'intercultura. Pisa: ETS, pp. 91-100.

Sikkens E., Sieckelinck S., Van San M., Winter M., de (2017): Parental Influence on Radicalization and De-radicalization According to the Lived Experiences of Former Extremists and their Families. Journal for Deradicalization, n. 12, pp. 192-225.

Tarabusi F. (2010): Adolescenti stranieri e sguardi di genere. Un approccio antropologico. In C. Gamberi, M.A. Maio, G. Selmi (a cura di): Educare al genere. Riflessioni e strumenti per articolare la complessità. Roma: Carocci, pp. 99-114.

Ulivieri S. (2017): Genere etnia e società. Pedagogia Oggi. n.1, pp. 9-16.

UNESCO (2015): Educazione alla cittadinanza globale: temi e obiettivi di apprendimento. https://unesdoc. unesco.org/ark:/48223/pf0000261836 (data di ultima consultazione: 12.02.21).

UNESCO (2016): The Global Counter-Terrorism Forum (GCTF; https://www.thegctf.org/Portals/1/ Documents/Toolkit-documents/English-The-Role-of-Familes-in-PCVE.pdf; data di ultima consultazione: 15.02 .21$)$.

UNESCO (2019): Guida per insegnanti sulla prevenzione dell'estremismo violento (https://www.cci.tn.it/ content/download/142458/1925383/version/2/file/Guida+per+insegnanti+sulla+prevenzione+de $11 \% 27$ estremismo+violento.pdf; data di ultima consultazione: 15.02.21).

UNESCO (2019): Prevenire l'estremismo violento attraverso l'educazione. Guida per amministratori pubblici (http://unescoblob.blob.core.windows.net/pdf/UploadCKEditor/Prevenirelestremismoviolento attraversoleducazione.Guidaperamministratoripubblici\%201.pdf; data di ultima consultazione: 15.02.21).

\section{Riferimenti sitografici}

https:/www.cci.tn.it/content/download/142458/1925383/version/2/file/Guida+per+insegnanti+sulla+ prevenzione+dell\%27estremismo+violento.pdf (data di ultima consultazione: 15.02.21).

https://ec.europa.eu/home-affairs/sites/homeaffairs/files/what-we do/networks/radicalisation_awareness_network/docs/manifesto-for-education-empowering-educators-and-schools_en.pdf (data di ultima consultazione: 15.02.21).

https://euroclio.eu/wp-content/uploads/2016/03/Manifesto-for-Education-Empowering-educators-andschools.pdf (data di ultima consultazione: 15.02.21).

https://www.europarl.europa.eu/EPRS/EPRS-Briefing-581955-Radicalisation-gender-perspective-revFINAL.pdf (data di ultima consultazione: 26.02.21).

https://www.osce.org (data di ultima consultazione: 20.02.2021).

https://www.thecommunityproject.eu (data di ultima consultazione: 10.11.21).

https://www.thegctf.org/Portals/1/Documents/Toolkit-documents/English-The-Role-of-Familes-inPCVE.pdf (data di ultima consultazione: 15.02.21).

https://ufmsecretariat.org/womens-rights-human-rights-mariya-gabriel/ (data di ultima consultazione: 15.02.21).

http://unescoblob.blob.core.windows.net (data di ultima consultazione: 15.02.21).

https://unesdoc.unesco.org (data di ultima consultazione: 15.02.21).

https://unric.org/it/agenda-2030/(data di ultima consultazione: 15.02.21). 
